Giovanna Martino Piccolino

\title{
La regola educativa di S. Gerolamo: esempi di institutio christiana nell'Epistolario geronimiano (Ep. 107 e 128)
}

\section{Hieronimovo vzgojno pravilo: institutio christiana $v$ Hieronimovih pismih (Ep. 107 in 128)}

The Educational Rule of St. Jerome: Examples From in-
stitutio christiana in His Epistolography (Ep. 107 in 128)

Riassunto: Le epistole ,pedagogiche' di Gerolamo (Ep. 107 e 128; la prima scritta tra il 400 e il 401, la seconda tra il 412 e il 413) costituiscono un interessante e prezioso documento della paideia cristiana: dispensando consigli, l'autore delinea curricula di apprendimento, ereditati dalla cultura romana (in particolare da Quintiliano) e metodi d'insegnamento ispirati dalla Sacra Scrittura. Gerolamo afferma che l'esempio dei genitori è più efficace di qualsivoglia regola o precetto. È necessario per i fanciulli apprendere sotto forma di gioco. L'educazione femminile, con l'affermarsi del Cristianesimo, diviene un tema fondamentale della speculazione pedagogica: nel testo un confronto con altri autori cristiani, come Gregorio di Nissa, sull'educazione delle vergini è supportato da opportuni rimandi testuali.

Parole chiave: educazione cristiana, famiglia romana, figlia, educazione della vergine, gioco, la donna nella cultura greco-romana, docenti, discenti.

Povžetek: Tako imenovani ,pedagoški' pismi sv. Hieronima (Pismo 107 in Pismo 128; prvo napisano leta 401/402 in drugo leta 412/413) pomenita zanimiv in dragocen dokument tistega, kar imenujemo krščanska paideia; avtor svetuje, začrta curricucula učenja, kakor nam jih je predala rimska kultura (predvsem Kvintilijan), in metode poučevanja, ki jih je navdihnilo Sveto pismo. Hieronim zatrjuje, da je zgled staršev veliko bolj uspešen kakor kakršnokoli pravilo ali zapoved. Za otroke je prav tako nujno, da sprejemajo pouk skozi igro. Vzgoja žensk je z uveljavitvijo krščanstva postala temeljna tema pedagoškega premisleka, zato je tema vzgoje devic $v$ članku obravnavana tudi v povezavi z drugimi krščanskimi avtorji, z Gregorjem iz Nise na primer. 
Ključne besede: krščanska vzgoja, družina v Rimu, hči, vzgoja devic, igra, ženska v grško-rimski kulturi, učitelji, učenci

Abstract. The ,pedagogical' epistles by Jerome (Ep. 107 and 128; the former written in 400-401 and the latter in 412-413) are an exciting and precious document from Christian paideia: giving advice, the author gets Scholarship curricula inherited from Roman culture (mainly from Quintilian) and the teaching methods provided by holy Bible. Jerome wrote that parents' example is more instructive than a lot of rules or precepts. Then children must learn to play. Woman's education, in Christianity, becomes a fundamental subject in the pedagogical reflection: in this text, comparison with other Christian authors, for example, gratia Gregorius Nyssenus, about virgins' education is supported by textual references.

Keywords: Christian education, Roman family, daughter, education of virgins, play, women in Greek and Roman society, students and teachers

\section{La cultura greco-romana e la predicazione di Gesù}

Nella graziosa novella di Gige e Candaule, ${ }^{1}$ Erodoto afferma che »con lo spogliarsi delle vesti la donna si spoglia anche del pudore : ${ }^{2}$ benché presso i barbari la nudità fosse disonorevole anche per l'uomo, è certamente da rilevare, come ha scritto lo Jaeger (2018, 1104-1110), un notevole cambiamento di sentire prodottosi ad Atene rispetto alla donna dall'età periclea ai primi decenni del IV secolo, quando Platone scrive la Politèia. In essa, infatti, il filosofo ateniese dedica attenzione alla formazione della donna aristocratica, futura consorte del guerriero, la quale dovrebbe essere cooperatrice nell'ufficio di lui: se comune dovrà essere il compito, tali dovranno necessariamente essere anche educazione e formazione. La donna aristocratica, pertanto, educata alla musica e alla ginnastica, non avrebbe dovuto vergognarsi di svestirsi nei ginnasî, perché insieme col marito guerriero anziché dell'himation si sarebbe vestita di areté. ${ }^{3}$ L'attuazione del programma di una formazione culturale unica per uomo e per donna avrebbe rafforzato, quindi, secondo Platone, l'unità dello stato ideale da lui ben delineato nel noto dialogo, conferendo alla classe dirigente quella superiorità richiesta dal compito. Occorre, infine, notare che anche in un tale progetto la donna non è destinataria di un programma educativo volto a coglierne e potenziare le peculiarità di genere, mentre il riconoscimento più elevato che le si riesca a concedere consiste nell'essere uguagliata al genere maschile (Cantarella 2010, 93-95).

1 Ringrazio il Preside del Pontificium Institutum Altioris Latinitatis, Prof. Don Miran Sajovic SDB, per il cordiale incoraggiamento. Resta, tuttavia, solo mia la responsabilità di eventuali errori o imperfezioni.

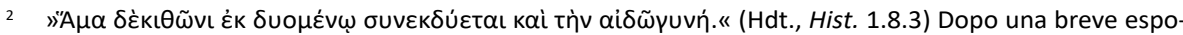
sizione sulle origini mitologiche del conflitto Europa-Asia, dalle quali Erodoto prende le distanze, l'autore introduce Creso, con il quale ha inizio il lógos sulla Lidia, fino al regno di Gige che soppianta Candaule perché indotto dalla moglie di questi a scegliere tra la morte sua o del marito, dopo che il sovrano aveva consentito a Gige di ammirarla nuda a sua insaputa. (Cassola 1984, 84-91)

3 Cf. PI., R. 452e-456c (Sartori, Vegetti e Centrone 2003, 304-315). 
Diversa è la situazione a Roma, dove l'istruzione non è preclusa alle ragazze, in particolare alle rampolle di famiglie gentilizie: la donna, quale mater educatrix, è depositaria del compito di trasmettere il mos maiorum ai figli, di provvedere alla loro educazione, almeno fino all'età di sette anni. II suo bagaglio culturale deve essere speso prevalentemente in ambito privato, evitando pubbliche esibizioni come simbolo di emancipazione e di superiorità rispetto all'uomo. Il curriculum scolastico prevede una prima istruzione elementare detta ludus litterarius, dai sette ai dodici anni circa, con la compresenza di ragazzi e ragazze: è prevista la lettura, la scrittura e il calcolo sotto la guida del litterator o ludi magister. Segue verso i dodici anni, in classi distinte tra maschi e femmine, l'insegnamento secondario, ovvero la scuola del grammaticus che insegna in prima istanza la lingua (ossia valore fonetico, declinazione, coniugazione, ritmo dei versi, parti di un discorso), in seconda l'uso corretto della lingua parlata e scritta (con l'introduzione degli autori e di esercitazioni sullo stile).

Ai soli maschi, in genere, è riservato l'accesso alla scuola di retorica, verso i quindici o i sedici anni: il rhetor, oltre a insegnare le regole del discorso, dedica attenzione allo studio del diritto insieme col magister iuris, nel quadro di una più ampia formazione filosofica (Lanfranchi e Prellezo 2008, 150-164).

La diffusione della predicazione di Gesù, nella prima età imperiale, s'innesta in una cultura ebraica dove il ruolo femminile assume funzioni nettamente subordinate alla volontà maschile, ${ }^{4}$ mentre per il mondo greco-romano non è possibile delineare un quadro univoco. In Grecia come a Roma, infatti, non mancano discriminazioni, tuttavia nei ceti sociali più elevati dell'Impero è possibile trovare donne emancipate, oppure (fenomeno del tutto assente in Palestina) la presenza di sacerdotesse nei culti pagani. ${ }^{5}$ Le donne nei Vangeli canonici, invece, hanno sempre ruoli ben definiti: alcune che procurano assistenza, anche mediante le proprie sostanze (LC 8,1-3), l'emorroissa, considerata impura, che sperimenta la guarigione immediata (Mt 9,20-22), la samaritana che, da una condizione poco rispettabile, diviene valida testimone per la sua città (Gv 4,1), la Maddalena tenace nella fedeltà al Maestro e Salvatore (Mc 16,9), Marta e Maria di Betania, dedite rispettivamente al servizio e all'adorazione del Maestro. Sono esse solo alcuni esempi della grande attenzione e della benefica cura spirituale di cui sono destinatarie le donne durante il ministero di Cristo (Fabris 1987, 209-222; Mattioli 1992; Marucci 1976, 273-296; Martino 2010, 615-624).

Filone spesso afferma l'inferiorità della femmina rispetto al maschio e, nella sfera umana, della donna rispetto all'uomo. La donna è debole, nata per essere ingannata, poco incline alla riflessione (Phil., Quaest. in Gen. 1.46). Il peccato, inoltre, è conseguenza della debolezza femminile (Phil., Quaest. in Gen. 3.3).

5 Basti pensare, a mo' di esempio, all'insigne ruolo delle Vestali a Roma, a partire dall'età arcaica: le giovani che venivano scelte per il culto di Vesta erano bambine tra i sei e i dieci anni, selezionate grazie ai requisiti delle loro famiglie d'origine. Ancora Prudenzio in Contra Symmachum 2.1066 dirà: "podii meliore in parte sedentes", riferendosi al fatto che assistevano alle rappresentazioni pubbliche, non relegate in summa cavea, come le altre donne, ma sedute nelle prime file, tradizionalmente riservate ai senatori. (Orlandi 1995-1996, 359-371) 


\section{Dall'età apostolica al IV secolo}

In età apostolica alcuni scritti sono rivolti indistintamente a fratelli e sorelle: I'Epistola di Barnaba si rivolge a "figli e figlie ${ }^{6}{ }^{6}$ mentre Adolf von Harnack ha descritto come le donne abbiano avuto fin dall'inizio un posto di rilievo accanto ai grandi maestri, anche nella diffusione della cultura cristiana di tipo scolastico o intellettuale (1906, 406-419). ${ }^{7}$ Taziano, ad esempio, esalta le figure femminili che "si

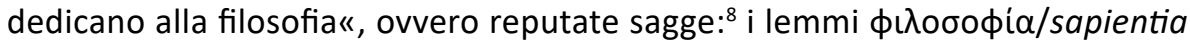
cominciano nel II secolo ad assumere l'accezione di "percorso interiore verso il superamento della dimensione terrena ${ }^{9}{ }^{9} \mathrm{e}$, a partire dal IV secolo, in concomitanza con l'evoluzione dell'ambito semantico del termine, che tende a lambire la realtà degli asceterî e dei monasteri, si profila un nuovo progetto pedagogico, specificamente rivolto alle donne da parte dei Padri, sia in Oriente, sia in Occidente, che consenta alla donna cristiana di anelare a un nuovo paradigma di santità, mediante una vita di ascesi, di preghiera e di continenza. ${ }^{10}$ Questo prodotto peculiare della cultura cristiana del IV secolo, che rappresenta, senza dubbio, un'evoluzione morale-speculativa rispetto alla coeva temperie storica ellenistico-giudaica ${ }^{11}$ eredita, tuttavia, sia dalla tradizione ebraica, sia da quella romana la centralità del ruolo della famiglia nella trasmissione dei valori. ${ }^{12} \mathrm{~A}$ tal proposito, un esempio illuminan-

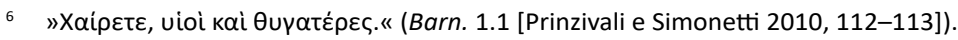

7 Per un approfondimento sulle donne cristiane, partecipi della cultura, in qualità di allieve, collaboratrici o protettrici di intellettuali cristiani cf. Mazzucco 1989, 10-16.

8 " $/$ /.../ affinché /.../ sotto i vostri occhi non deridiate quelle donne che presso di noi si dedicano alla filosofia. /.../ Tutte le nostre donne vivono nella continenza e le fanciulle intorno alla conocchia rivolgono espressione a Dio con più ardore d’ogni vostra fanciulla.« (Tat., Orat. [Burini 2000, 230-231])

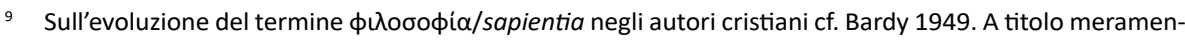
te esemplificativo vale la pena ricordare le parole di Socrate di Costantinopoli, quando descrive la bio-

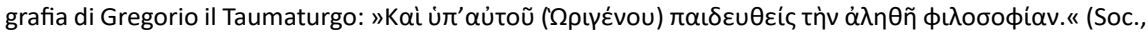

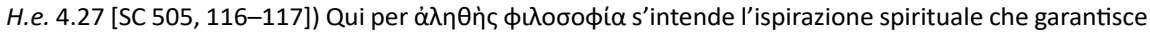
la veritiera interpretazione delle Scritture.

10 Sulla novità del progetto educativo dei Padri rivolto al genere femminile cf. Milazzo 2002; sulla funzione profetica e sul diaconato femminile all'interno della comunità cristiana cf. Hauser-Borel 2000; Uglione 1989; Gryson 1974; Mara 1981; Rousselle 2000, 143-165.

11 Si ricorda, tuttavia, in ambito romano, la figura di Musonio Rufo, filosofo stoico, vissuto nel I secolo e maestro di Epitteto, nelle cui pagine a noi pervenute è del tutto assente l'immagine tradizionalmente negativa della donna: nella Diatriba IV afferma che figli e figlie vanno educati allo stesso modo perché sia gli uni, sia le altre diventino virtuosi. Di interesse è anche la Diatriba III per il tema del rapporto delle donne con la cultura che si intitola Del fatto che anche le donne debbano accostarsi alla filosofia. Cf. Ramelli, llaria, ed. 2001. Musonio Rufo: Diatribe, Frammenti e Testimonianze. Milano: Bompiani. Sul caso di Ipazia di Alessandria cf. Cameron 2013, 65-82; Ronchey, 2011; Ronchey 2014, 135-169.

12 »/.../ maiores nostri /.../ domum pusillam rem publicam iudicaverunt. « (Sen., Ep. 47.14 [Barone 1989, 224-227]) Questa espressione corrisponde alla struttura piramidale della familia Romana, dove i ruoli dei membri sono ben definiti e il cui ordine è garantito dalla presenza del pater familias. La familia romana è un organismo in sé autosufficiente e che si autodetermina in ambito educativo, essendo questa la dimensione sociale che maggiormente le compete. (Frasca 1991, 9-11, 14-15) I rapporti affettivi competevano maggiormente al ruolo materno, mentre il padre era soprattutto un severo custode della tradizione. Utile, in proposito la testimonianza ciceroniana: „Prima societas in ipso coniugio est, proxima in liberis, deinde una domus, communia omnia; id autem est principium urbis et seminarium rei publicae." (Cic., De off. 1.54 [Narducci e Resta Barrile 2004, 122-123]) Sul ruolo attribuito alla famiglia nella trasmissione dei valori, come già avveniva nel mondo romano e giudaico cf. Marrou 2016, 642-646. Diversa è la famiglia cristiana costituita da due coniugi ferventi nella fede: è il caso delineato 
te è costituito dalla Vita di S. Macrina, scritta con devozione fraterna da Gregorio di Nissa: ${ }^{13}$ l'educazione impartita da Emmelia, madre di Macrina nonché figlia di Macrina senior, la quale era stata discepola di Gregorio Taumaturgo, ${ }^{14}$ indirizza la giovane verso un modello di santità plasmato sull'esempio di quello proposto da Atanasio nella sua Vita di Antonio. L'opera, scritta su richiesta del fratello Basilio, segue cronologicamente la stesura del Trattato sulla verginità, ${ }^{15}$ realizzando così una sorta di ideale continuità tra la riflessione teorica e la sua esemplificazione sulla vita virtuosa, alla quale il Nisseno intende invogliare il lettore. ${ }^{16}$

\section{San Gerolamo e l'educazione delle fanciulle}

Anche Gerolamo recepisce l'ideale ascetico della Vita di Antonio, ${ }^{17}$ che ha, secondo gli studiosi, ${ }^{18}$ un ruolo determinante nella formazione del monachesimo occidentale: grazie alla mediazione del patriarca alessandrino Atanasio (nonché di varî monaci dai quali sarebbe stato accompagnato), rifugiatosi dapprima a Roma, poi a Milano e infine ad Aquileia in seguito alle ostilità degli ariani a partire dal 339, I'ideale ascetico di origine egiziana si diffonde rapidamente in Occidente. ${ }^{19}$ In particolare, le testimonianze letterarie parlano di un ascetismo al femminile, cui sono

da Tertulliano in Ad uxorem: "Quale iugum fidelium duorum unius spei, unius voti, unius disciplinae, eiusdem servitutis. Ambo fratres, ambo conservi; nulla spiritus carnisve discretio, atquin vere duo in carne una. Ubi caro una, unus et spiritus: simul orant, simul volutantur, simul ieiunia transigunt, alterutro docentes, alterutro exhortantes, alterutro sustinentes." (Tert., Ad Uxor. 2.8.7 [Isetta et al. 2008, 318-321]). Una donna timorata di Dio, invece, curerà di piacere solo al proprio marito, come afferma sempre Tertulliano: "Sancta femina sit naturaliter speciosa, non adeo sit occasio. Certe si et fuerit, non ignorare, sed etiam impedire debet. Quasi gentili bus dicam, gentili et communi omnium praecepto alloquens vos: solis maritis vestris placere debetis. "(Tert., Cult. fem. 2.3.4 [Isetta et al. 2008, 376-377]).

13 Cf. La Vita di S. Macrina di S. Gregorio di Nissa (Giannarelli 1988). Un ottimo contributo esegetico è quello offerto dall'introduzione in Grégoire de Nysse, Vie de sainte Macrine, dove si legge: "d'une éducation entièrement domestique, que se réserve la mère de Macrine. "(Gr. Nyss., V. Macr. 3.6 [SC 178, 51])

14 Macrina senior è definita da Basilio come colei che trasmette ai nipoti la dottrina di Gregorio Taumaturgo (Bas., Ep. 204.6), mentre nell'Ep. 223.3 scrive di aver ricevuto la prima idea di Dio da sua madre e dalla nonna.

15 II Trattato sulla Verginità fu scritto nel 371, mentre la Vita di S. Macrina nel 380.

16 Evidente influsso esercitò la Vita di Macrina nella Patristica e in particolare su Geronzio, autore della Vita di Melania, la ricca matrona romana, che dopo la morte dei figli, insieme con il marito, decide di liquidare i beni, di vivere in castità e di viaggiare in Africa, Terra Santa ed Egitto, fondando monasteri e dedicandosi a una rigidissima ascesi. Cf. Gorce, Denys, ed. 1962. Vie de sainte Mélanie. SC 90. Paris: Cerf.; Coco, Lucio, trad. 2013. Geronzio: Vita latina di Santa Melania. Roma: Città Nuova. Delle due versioni quella greca dovrebbe essere l'originale rispetto a quella latina ed è storicamente affidabile e importante per le informazioni sullo sviluppo dell'ascesi cristiana. (Perrone 1980; Giardina 1994)

17 Evagrio di Antiochia la tradusse in latino prima del 375, mentre un'altra traduzione latina anonima, meno letteraria, è più antica. Entrambe traduzioni suscitarono in Occidente interesse autentico per il fenomeno del monachesimo, costituendo, inoltre, un modello per la letteratura agiografica latina (cf. Ruggiero, Fabio, ed. 2003. Sulpicio Severo: Vita di Martino. Bologna: Edizioni Dehoniane Bologna.

18 Il ruolo dell'Oriente nell'impulso al monachesimo occidentale si espletò, con rapidità, mediante la circolazione di traduzioni provenienti dal milieu egiziano (e cappadoce). Oltre la Vita di Antonio si diffusero le regole basiliane e la Historia monachorum, libera traduzione della Storia dei monaci d'Egitto, di Rufino di Aquileia, nonché il corpus delle regole di Pacomio, tradotte da Gerolamo nel 404. Per un ragguaglio esaustivo sull'argomento cf. Vecoli 2015.

19 Lo stesso Gerolamo ce ne dà notizia in Ep. 127.5. 
connesse le origini del monachesimo latino in Terrasanta, di antica tradizione e sovente rappresentato da personaggi legati all'aristocrazia di Roma.

Mario Maritano, nel suo pregevole contributo alla comprensione della figura del maestro nella pedagogia geronimiana (2012), evidenzia l'importanza delle Epp. 107 e $128,{ }^{20}$ scritte rispettivamente nel $400-401$ e nel 412-413, quali documenti illuminanti ai fini di una certa e articolata educazione cristiana delle virgines. ${ }^{21}$ Non si può non convenire con l'illustre patrologo su questo punto, tenuto conto del fatto che in esse Gerolamo trae dalla Bibbia motivi di intervento e di impegno, ispirandosi anche a pratiche e programmi scolastici ereditati dalla cultura romana e, in particolare, da Quintiliano. In sintesi, la tradizione classica viene in queste lettere rimodulata e rinnovata alla luce della visione cristiana della vita.

Nell'Epistula 107 (ad Laetam de institutione filiae), alla nuora di Paola, ${ }^{22}$ che ha dato alla luce una fanciulla (anch'essa di nome Paola, in ossequio alla di lei $a v a$ ) facendo voto di consacrarla alla vita religiosa, la quale chiede come educare al meglio cristianamente la bambina, lo Stridonense risponde che la virgo, destinata ad essere »Tempio del Signore" e »consacrata a Cristo« dovrà ispirare la sua educazione e la vita, in ogni suo aspetto, al messaggio evangelico. Inoltre, avrà anche

20 Il testo latino utilizzato è ripreso per Ep. 107 e Ep. 128 dall'edizione di Labourt (2002a; 2002b), invece la traduzione e di Cola (1996). Resta tuttavia proficua la consultazione dello spicilegio (Moreschini 2000, 434-469). Per una bibliografia specifica sulle Ep. 107 e 128, cf. Labanca 1901; Lanfranchi 2008.

21 Sul problema della verginità Gerolamo si era scagliato contro il monaco Gioviniano nel Contra lovinianum (PL 25, 211-338). Costui, giunto a Roma forse dal nord, aveva spinto al matrimonio delle vergini consacrate, suscitando lo sferzante intervento di Gerolamo da Betlemme, mediante attacchi ad personam, con l'impiego a dismisura di testimonianze bibliche, nonché con la ripetizione di argomenti attinti anche da Tertulliano. A Roma gli amici di Gerolamo vennero a trovarsi in una situazione imbarazzante, per i toni eccessivi che aveva assunto la polemica con l'intervento dello Stridonense, tanto da spingere l'amico Pammachio (genero di Paola) a ridurre il numero di copie dell'opera in circolazione. L'epistola geronimiana 49, Apologeticum ad Pammachium, ben testimonia la cooperazione in prima linea dell'amico romano: "Ego, si bene problematis memini, inter lovinianum et nos ista contentio est, quod ille exaequet virginitati nuptias, nos subiciamus; ille vel parum vel nihil, nos multum interesse dicamus. Denique idcirco te post dominum faciente damnatus est, quod ausus sit perpetuae castitati matrimonium comparare." (Hier., Ep. 49.2 [Moreschini e Palla 2000, 266-267]) Nel 390, o poco dopo, la fazione favorevole al monachesimo e in particolare Pammachio indussero la condanna di una decina di partigiani di Gioviniano (cf. Siricio, Ep. 7 [PL 13, 1168-1172]). Ambrogio e un suo sinodo espressero formale condanna di Gioviniano (PL 16, 1124-1129). Agostino più tardi interverrà sull'argomento con il De sancta virginitate, in difesa della verginità, contro Gioviniano e Vigilanzio, ove oltre lodare suddetta virtù ammonirà le vergini perché non insuperbiscano. Cf. Recchia 1966; Duval 2003.

22 Sulla nobildonna romana Paola, la testimonianza più completa lasciata da Gerolamo è certamente I'Epitaphium Sanctae Paulae, che figura come Ep. 107, dedicata alla figlia Eustochio, e destinata, certamente, a un pubblico più vasto. L'intento dichiarato (cap. 2) dell'autore è quello di rendere testimonianza alla vita esemplare della nobildonna. Segue la narrazione del matrimonio e, con la morte del marito, di una vita ascetica che si traduce in grande carità e liberalità verso il prossimo. In seguito all'incontro con alcuni vescovi orientali (giunti a Roma per una sinodo), Paola si imbarcherà per l'Oriente, insieme con la figlia Eustochio; giunta ad Antiochia, da dove secondo studi autorevoli (Cavallera 1922, 123) insieme con Gerolamo e altri compagni, intraprese il viaggio in Terrasanta, per poi muovere alla volta dell'Egitto, culla di asceti e anacoreti. L'umiltà, unita alle pratiche ascetiche e penitenziali, nel quadro di una castità irreprensibile, delineano il ritratto di una donna integerrima, severa nella disciplina prima con sé stessa, esempio di umiltà, tenacia e liberalità che si manifestano soprattutto nell'organizzazione della vita monastica a Betlemme. Concludono l'epitaphium due poemetti epigrafici, incisi sulla sua tomba, che menzionano i cinque anni di vita ascetica a Roma e i venti a Betlemme, in osservanza anche alla gran dama romana, rampolla degli Scipioni e dei Gracchi, secondo un gusto poetico di ascendenza damasiana. Cf. Mohrmann 1975. 
Maria, madre di Gesù, come suo modello e a lei dovrà ispirarsi nei comportamenti: da altri Padri della Chiesa, come Ambrogio, ${ }^{23}$ Maria è espressamente indicata quale magistra per le vergini, mentre San Paolo, nella medesima epistola, viene definito praeceptor da Gerolamo per gli opportuni consigli dispensati ai cristiani.

In senso primario e manifesto, maestri sono gli stessi genitori della fanciulla. Con chiarezza rivolgendosi alla madre, poi al padre Gerolamo scrive: „Te habeat magistram, te rudis miretur infantia. Nihil in te et in patre suo videat, quod si fecerit, peccet. Mementote vos parentes virginis et magis eam exemplis docere posse quam voce.« (Hier., Ep. 107.9) ${ }^{24}$

In primo luogo, il nostro Autore intende porre l'accento sull'insegnamento morale e sull'esempio; in secondo luogo, nel più specifico ambito scolastico, egli richiama come fondamentale la corretta pronuncia delle parole:

»Unde et tibi est providendum, ne ineptis blanditiis feminarum dimidiata dicere filiam verba consuescas et in auro atque in purpura ludere, quorum alterum linguae, alterum moribus officit, ne discat in tenero, quod ei postea dediscendum est. Graccorum eloquentiae multum ab infantia sermo matris scribitur contulisse, Hortensiae oratio in paterno sinu coaluit. Difficulter eraditur, quod rudes animi perbiberunt. Lanarum conchyliaquis in pristinum candorem revocet? Rudis testa diu et saporem retinet et odorem, quo primum imbuta est.« (Ep. 107.4) $)^{25}$

È evidente anche in questo caso che la madre, in particolar modo, a continuo contatto con i figli piccoli, risulti la prima magistra dicendi. Questo è quanto afferma, ad esempio, Cicerone riferendosi ai Gracchi, figli di Cornelia: "Sed magni interest quosquisque audiat cotidie domi, quibuscum loquatur a puero, quemadmodum patres, paedagogi, matres etiam loquantur. Legimus epistulam Corneliae matris Gracchorum; apparet filios non tam in gremio educatos quam in sermone matris." (Cic., Brut. 210-211) ${ }^{26}$ La neglegentia parentum, per contro, fu conside-

23 „Il primo stimolo dell'apprendimento è costituito dalla nobiltà del maestro. Che cosa c'è di più nobile della madre di Dio?« (Ambr., Virg. 2.2.7 [SAEMO 14/1, 168])

24 „Te abbia come sua maestra, te ammiri la sua infanzia inesperta. In te e in suo padre non veda cosa che, da lei imitata, la porti a peccare. Ricordatevi che siete i genitori di una vergine e che potete esserle di insegnamento più con l'esempio che con le parole."

25 „Perciò devi anche provvedere a che tua figlia, per le insulse moine delle donne, non prenda l'abitudine di pronunciare le parole a metà e di dilettarsi nell'oro e nella porpora (la prima cosa nuoce alla lingua, la seconda ai costumi) e a che non impari in tenera età cose che dovrebbe poi disimparare. Sta scritto che all'eloquenza dei Gracchi contribuì molto, fin dall'infanzia, il modo di parlare della madre e che l'abilità oratoria di Ortensia si formò in seno al padre. Si cancella con difficoltà ciò che menti vergini hanno assorbito. Chi può ricondurre all'antico candore le lane tinte di porpora? Un'anfora nuova mantiene a lungo il sapore e l'odore di ciò che ha contenuto la prima volta."

26 „Ma importa molto chi uno senta parlare tutti i giorni in casa, con chi parli fin da fanciullo, in che modo parlino i padri, i pedagoghi e anche le madri. Se leggiamo le lettere di Cornelia madre dei Gracchi, appare evidente che i suoi figli sono stati educati non tanto nel grembo quanto nell'idioma della madre." (Narducci 1995, 276-279) Le medesime osservazioni vengono avanzate da Cicerone a proposito dei Catuli, figlio e padre (Cic., Brut. 133), e di Scipione l'Emiliano, »usu et domesticis praeceptis« (Scip., Rep. 1.22.36 [Ferrero e Zorzetti 1975, 103-104]). 
rata una delle cause principali della corruzione dell'eloquenza in età imperiale e, in generale, della decadenza dei costumi: ${ }^{27}$ non solo i genitori, in età tardo-repubblicana, avevano smesso di occuparsi personalmente dell'educazione dei figli, ${ }^{28} \mathrm{ma}$ con il loro comportamento li inducevano alla lascivia e alla dicacitas.

Nell'epistola 128, che l'autore definisce col diminutivo epistulium, quasi a evidenziarne il tono confidenziale, indirizzata a Gaudenzio, sull'educazione dell'infante Pacatula, oltre a rimodulare concetti espressi nella lettera precedente, si accentuano le peculiarità dell'ambiente nel quale la giovinetta va educata, sereno e gioioso, che sappia trasmettere l'amore per la cultura, senza indulgere a espressioni disoneste o a compagnie maschili. Oltre al lavoro intellettuale, la fanciulla dovrà abituarsi anche ai lavori manuali, caratteristici della donna romana, in particolare la tessitura, insieme col lavoro di confezionare vestiti, occupazione costante nell'ascetismo e nel monachesimo femminile: »Interim et tenero temptet pollice fila deducere; rumpat saepe stamina, ut aliquando non rumpat « (Ep. 128.1); $;^{29}$ in tal modo si rifugge l'ozio (consuetudinario nella nobiltà), e si produce un'opera compiuta con le proprie mani. Gerolamo richiede anzitutto che le persone, le quali dovranno accudire all'educazione e all'istruzione della bambina siano di moralità provata, tuttavia che ella eviti di legarsi morbosamente a qualche domestica: la governante modello deve guidare la fanciulla in una formazione monastica, con scanditi orari di preghiera diurna e notturna (107.9). Riferendosi poi al maestro, Gerolamo ne riporta tre qualità da richiedersi: età, condotta ineccepibile, sapere. Erano queste virtù legate a valori umani apprezzati anche dai gentili: ${ }^{30}$ l'azione dei maestri a Roma fu sempre risultante della diretta influenza paterna, garante di moralità, severità e competenza.

Lo Stridonense, concludendo la lettera a Leta, le suggerisce di affidare la figlia al monastero di Betlemme, data l'impossibilità di allevare convenientemente una vergine nella mondana società contemporanea. Egli stesso si offre come possibile maestro e precettore di Paola, la quale circa quindici anni più tardi, sarà inviata nel monastero di Betlemme, accanto alla nonna Paola e alla zia Eustochio. In esordio, richiamando l'esempio di Aristotele, che fu maestro di Alessandro Magno, l'Autore aveva dichiarato la responsabilità di pari grado nell'educare una nobile vergine (Hier., Ep. 107), sposa del Signore.

Da Rufino apprendiamo che Gerolamo avviò una scuola nel monastero di Betlemme, insegnando ai giovani studenti la grammatica e la lettura di autori classici latini, in particolare Virgilio, i comici, i lirici e gli storici (Ruf., Apol. adv. Hier. 2.11).

27 Espressione massima di questo disinteresse era il rifiuto della paternità che creava in Grecia e a Roma gravi problemi demografici come riporta Polibio (Nicolai 1998, 344-345).

28 Si tratta di un'usanza contraria all'antica educazione, secondo quanto testimoniato in Plutarco: »/.../ Pensava non fosse dignitoso, come egli stesso diceva, che uno schiavo potesse rimproverare suo figlio nel caso che fosse lento nell'apprendere, né che fosse debitore a uno schiavo di una cosa così grande come l'istruzione /.../.« (Plu., Cat. Ma. 20.5-6 [Traglia 1992, 636-637])

29 „Nel frattempo col suo tenero pollice provi a sconocchiare; spezzerà sovente il filo, ma è così che impara a non spezzarlo più."

30 La mollis educatio dei suoi tempi, secondo Quintiliano, spinge i giovani inconsapevolmente ai vizî ( $\mathrm{Pi}$ scitelli 2001, 34-35). 
Egli avverti la necessità di formare le giovani generazioni e »dar loro le armi per difendere le loro convinzioni: questo può avvenire solo con l'apprendimento dei grandi modelli che la letteratura fornisce. /.../ Anche nel problema della scuola egli è romano: insegna secondo il sistema scolastico romano, che non insegnava idee, ma come esprimere idee con proprietà e ricchezza; anche qui è filologo e letterato, non teologo.« (Grilli 2004, 312)

Infine, dalle epistole esaminate si possono dedurre gli ulteriori seguenti principî educativi enunciati: un ambiente sereno e gioioso stimola l'allievo a dare il meglio di sé stesso, dunque anche l'apprendimento delle lettere dell'alfabeto deve diventare un gioco. In secondo luogo, un clima di amore per la cultura, nello sforzo che essa implica, favorisce l'erudizione: lusus eruditio sit (Hier., Ep. 107.4). L'allieva dovrà, inoltre, preferire lo studio dei testi sacri, ad altri beni preziosi che pure si possono godere e deve dilettarsi nella lettura di trattati e opere di scrittori ispirati dalla fede.

Gerolamo scrive da cristiano per cristiani, pertanto il suo punto di riferimento primigenio è costituito, quanto ai contenuti, dalla Sacra Scrittura, nonché dalla tradizione classica romana, quanto alla forma. Qui la tradizione scolastica quintilianea si ammanta di un superiore rigore morale nell'essere ,donata' ai genitori di due virgines, destinate alla vita ascetica.

Con la scuola di Betlemme, in definitiva, lo Stridonense, già allievo del grammatico Elio Donato, fonda un'istituzione educativa ove il Cristianesimo diviene naturale erede della tradizione pedagogica del mondo antico, nella consapevole traditio della humanitas antica, quale presupposto ad una piena e consapevole accoglienza del dono di Dio.

\section{Abbreviazioni}

Ep. - Epistulae [Labourt 2002a; 2002b].

SAEMO - Sancti Ambrosii Episcopi Mediolanensis Opera.

SC - Sources Chrétiennes.

\section{Riferimenti bibliografici}

Fonti primarie

Barone, Caterina, ed. 1989. Seneca: Lettere a Lucilio. Milano: Garzanti.

Burini, Clara, ed. 2000. Gli Apologeti Greci. Roma: Città Nuova.

Ferrero, Leonardo, e Nevio Zorzetti, ed. 1974. Opere politiche e filosofiche di M. Tullio Cicerone. Torino: UTET.

Gori, Franco, ed. 1989. Opera omnia di sant'Ambrogio: Verginità e vedovanza. SAEMO 14/1. Milano: Biblioteca Ambrosiana; Roma: Città Nuova.
Labourt, Jérôme, ed. 2002a. Jérôme: Correspondance. Vol. 5, Lettres 46-59. Paris: Les Belles Lettres.

- - . 2002b. Jérôme: Correspondance. Vol. 1, Lettres 71-80. Paris: Les Belles Lettres.

Maraval, Pierre, ed. 1971. Grégoire de Nysse: Vie de sainte Macrine. SC 178. Paris: Cerf.

Mohrmann, Christine, A. A. R. Bastiaensen e J. W. Smith, ed. 1975. Hieronymi Epitaphium Sanctae Paulae. Trad. it. Luca Canali. Milano: Fondazione Lorenzo Valla; Arnoldo Mondadori Editore.

Narducci, Emanuele, e Anna Resta Barrile, ed. 2004. Cicerone: I doveri. Milano: BUR. 
Narducci, Emanuele, ed. 1995. Cicerone: Bruto. Milano: Rizzoli.

Pennacini, Adriano, ed. 2001. Quintiliano: Institutio Oratoria. Torino: Einaudi.

Périchon, Pierre, e Pierre Maraval, ed. 2006. Socrate De Constantinople: Histoire Ecclésiastique (Livres 4-5). SC 505. Paris: Cerf.

Prinzivalli, Emanuela, e Manlio Simonetti, ed. 2010. Seguendo Gesù: Testi Cristiani delle Origini. Vol. 2. Milano: Fondazione Lorenzo Valla/ Arnoldo Mondadori.

\section{Fonti secondarie}

Bardy, Gustave. 1949. Philosophie et Philosophe dans le Vocabulaire Chrétien des premiers siècles. Revue d'Ascétique et Mystique 25:97-108.

Cameron, Alan. 2013. The life, Work and Death of Hypatia. In: Michel Tardieu e Delphine Lauritzen, ed. Le voyage des légendes: Hommages à Pierre Chuvin, 65-82. Paris: CNRS,

Cantarella, Eva. 2010. L'ambiguo malanno. Milano: Feltrinelli.

Cassola, Filippo, ed. 1984. Erodoto: Storie. Milano: BUR.

Cavallera, Ferdinand. 1922. Saint Jérôme, sa vie et son ouvre. Vol. 2. Louvain; Paris: Champion.

Cola, Silvano, ed. 1996. San Girolamo: Lettere. Roma: Città Nuova.

Covolo, Enrico dal, e Isidoro Giannetto. 1998. Cultura e promozione umana: La cura del corpo e dello spirito nell'antichità classica e nei primi secoli cristiani; Un magistero ancora attuale? Troina: Oasi Editrice.

Duval, Yves Marie. 2003. L'affaire Jovinien: D'une crise de la société romaine à une crise de la pensée chrétienne à la fin du IV siècle. Roma: Institutum Patristicum Augustinianum.

Fabris, Rinaldo. 1987. La donna nel Nuovo Testamento. In: Renato Uglione, ed. Atti del Convegno nazionale di studi su La donna nel mondo antico, 209-222. Torino: Associazione italiana di cultura classica, Delegazione di Torino.

Frasca, Rossella. 1991. Donne e uomini nell'educazione a Roma. Firenze: La Nuova Italia.

Giannarelli, Elena, ed. 1988. S. Gregorio di Nissa: Vita di Macrina. Milano: Edizioni Paoline.

Giardina, Andrea. 1994. Melania, La Santa. In: Augusto Fraschetti, ed. Roma al femminile, 259285. Roma: Laterza.

Grilli, Alberto. 2004. Alla scuola di San Gerolamo. In: Gianpaolo Urso, ed. Dall'Adriatico al Danubio: L'Illirico nell'età greca e romana, 385-394. Atti del Convegno Internazionale: Cividale del Friuli, 25-26 settembre 2003. Pisa: Edizioni ETS.

Gryson, Roger. 1974. Il ministero della donna nella chiesa antica. Roma: Città Nuova.
Harnack, Adolf von. 1906. Missione e propagazione del cristianesimo nei primi tre secoli. Trad. it. P. Marrucchi. Torino: Bocca.

Hauser-Borel, Sylvie. 2000. Profetesse, martiri, testimoni di Cristo nella storia. In: Cettina Militello, ed. Profezia, modelli e forme nell'esperienza cristiana laicale, 125-154. Padova: CEDAM.

Isetta, Sandra, Sara Matteoli, Teresa Piscitelli e Valentina Sturli, ed. 2008. Tertulliano, Opere Catechetiche: Gli spettacoli; La preghiera; II Battesimo; La pazienza; La penitenza; Alla moglie; L'eleganza femminile. Roma: Città Nuova.

Jaeger, Werner. 2018. Paideia: La formazione dell'uomo greco. Trad. it. Luigi Emery e Alessandro Setti. Milano: Bompiani.

Labanca, Baldassarre. 1901. Le idee pedagogiche di S. Girolamo. Milano: Aliprandi.

Lanfranchi, Rachele, e Josè M. Prellezo. 2008. Educazione, scuola e pedagogia nei solchi della storia. Vol. 1, Dall'educazione antica al secolo del metodo. Roma: LAS.

Lanfranchi, Rachele. 2008. San Girolamo e l'educazione della donna. In: Rachele Lanfranchi e Josè M. Prellezo, ed. Educazione, scuola e pedagogia nei solchi della storia. Vol. 1, Dall'educazione antica al secolo del metodo, 180-187. Roma: LAS.

Mara, Maria Grazia. 1981. Le funzioni della donna nella chiesa antica. Rivista di pastorale liturgica 19 , no. 2:5-16.

Maritano, Mario, e Miran Sajovic, ed. 2012. Docere et Discere: La figura del Maestro nella formazione scolastica del mondo antico pagano e cristiano. Roma: Editrice LAS.

Maritano, Mario. 2012. II maestro nelle lettere pedagogiche di Girolamo (Epp. 107 e 128). In: Maritano e Sajovic 2012, 167-190.

Marrou, Henri-Irénée. 2016. Storia dell'educazione nell'antichità. Trad. it. Umberto Massi. Roma: Edizioni Studium.

Martino, Giovanna. 2010. II diaconato femminile nella chiesa primitiva: note di esegesi patristica. In: Diakonia, Diaconiae, Diaconato. Semantica e Storia nei Padri della Chiesa, 615-624. 38. Incontro di studiosi dell'antichità cristiana. Roma: Institutum Patristicum Augustinianum.

Marucci, Corrado. 1976. La donna e i misteri nella Bibbia e nella tradizione. Rassegna di teologia 17:273-296.

Mattioli, Umberto. 1992. La donna nel pensiero cristiano antico. Bologna: Marietti.

Mazzucco, Clementina. 1989. ,E fui fatta maschio': La donna nel cristianesimo primitivo (secoli I III). Firenze: Le Lettere.

Milazzo, Vincenza. 2002. Educare una vergine: Precetti e modelli in Ambrogio e Gerolamo. Ca- 
tania: Università di Catania.

Mohrmann, Christine, ed. 1975. In Memoria di Paola. In: Vite dei Santi: Vita di Martino; Vita di Sulpicio Severo; Vita di llarione; In memoria di Paola, 145-237. Trad. it. Luca Canali e Claudio Moreschini. Milano: Fondazione Lorenzo Valla; Arnoldo Mondadori Editore.

Moreschini, Claudio, ed. 1989. San Gerolamo: Lettere. Trad. it. Roberto Palla. Milano: BUR.

- - - 2000. San Gerolamo: Lettere. Trad. it. Roberto Palla. Milano: BUR.

Narducci, Emanuele, ed. 1994. Cicerone: Dell'oratore. Milano: BUR.

Nicolai, Roberto, ed. 1998. Polibio: Storie. Vol. 4. Roma: Newton.

Novembri, Valeria. 2005. L'educazione delle donne nel cristianesimo antico: fra modelli tradizionali e nuovi paradigmi. Storia delle donne 1:187-200.

Orlandi, Silvia. 1995-1996. Osservazioni prosopografiche sulle vestali. Rendiconti della Pontificia Accademia di Archeologia 68:359-371.

Perrone Lorenzo. 1980. La Chiesa di Palestina e le controversie cristologiche: Dal concilio di Efeso (431) al secondo concilio di Costantinopoli (553). Brescia: Paideia Editrice.

Piscitelli, Teresa, ed. 2001. Quintiliano: Institutio oratoria. Torino: Einaudi.

Recchia, Vincenzo. 1966. Verginità e martirio nei colores di S. Gerolamo. Vetera Christianorum 3:45-68.

Ronchey, Silvia. 2011. Ipazia: La vera storia. Milano: BUR.

- - 2014. Perché Cirillo assassinò Ipazia? In: A. Marcone, U. Roberto e I. Tantillo, ed. Tolleranza religiosa in età tardo antica. IV-V secolo: Atti delle giornate di studio sull'età tardo antica, 135171. Cassino: Edizioni Università di Cassino.

Rousselle, Aline. 2000. Donne cristiane e fondazioni testamentarie (III-IV secolo). In: Giuliana Lanata, ed. I/ tardo-antico alle soglie del Duemila, 143-165. Pisa: ETS.

Sartori, Franco, Mario Vegetti e Bruno Centrone, ed. 2003. Platone: La Repubblica. Roma; Bari: Laterza.

Traglia, Antonio, ed. 1992. Plutarco:Vite Parallele. Vol. 1. Torino: UTET.

Uglione, Renato, ed. 1989. Atti del I/ Convegno nazionale di studi su La donna nel mondo antico. Torino: Celid Editrice.

Vecoli, Fabrizio. 2015. II monachesimo antico. In: Emanuela Prinzivalli, ed. Storia del Cristianesimo. Vol. 1, L'età antica, 281-307. Roma: Carocci. 